\title{
REPARATIVE PHASE EVENTS ON PERIODONTAL DISEASE PROGRESSION: INTERPRETATION AND CONSIDERATIONS
}

\author{
KINA J.R. ${ }^{*}$, SUZUKI T.Y.U. ${ }^{2}$, KINA J. ${ }^{3}$, KINA M. ${ }^{4}$ AND KINA E.F.U. ${ }^{4}$ \\ ${ }^{1}$ Department of Surgery and Integrated Clinic, Araçatuba School of Dentistry, São Paulo State University - UNESP, Araçatuba, SP, Brazil. \\ 2Department of Dental Materials and Prosthodontics, Araçatuba School of Dentistry, São Paulo State University- UNESP, Araçatuba, SP, Brazil. \\ ${ }^{3}$ Department of Pediatric Dentistry and Public Health, Araçatuba School of Dentistry, São Paulo State University- UNESP, Araçatuba, SP, Brazil. \\ 4Private Practice, Araçatuba, SP, Brazil. \\ *Corresponding Author: Email- kinajr@hotmail.com
}

Received: June 15, 2013; Accepted: June 27, 2013

\begin{abstract}
Periodontal disease progress by destructive acute phases intercalated by reparative chronic phases. The aim of this study was to investigate the clinical and histological evidence of the periodontal disease reparative phase by analyzing bone wall conditions inside periodontal pockets and histologic images of periodontal pockets, identified in relevant publications. 81 patients with periodontitis, were randomly assigned into this study. Clinical and radiographic parameters were established to diagnose periodontal disease providing a sample of 133 diseased areas, which were treated by modified Widman flap. Documentation by digital photography were recorded in the surgery. Relevant publications showing histological images of periodontal pockets, were identified in Medline, PubMed and Google data base, were scanned and digitalized. All images obtained were evaluated and the presences of the reparative evidence in the zone around the underlying destroyed alveolar bone were critically analyzed. All periodontal bone defects, showed cortical bone reparations at different levels inside periodontal bone defects. All histologic images of periodontal pockets identified in relevant publications showed repaired gingival-attached connective tissue localized above underlying destroyed alveolar bone. All the evidences analyzed in this study suggested that periodontal disease is predominantly chronic, quiescent, showing reparative phases in different levels.
\end{abstract}

Keywords- Diagnosis, Evidence-based dentistry, Pathogenesis of periodontal disease, Periodontal surgery, Periodontal regeneration

Citation: Kina J.R., et al. (2013) Reparative Phase Events on Periodontal Disease Progression: Interpretation and Considerations. International Journal of Microbiology Research, ISSN: 0975-5276 \& E-ISSN: 0975-9174, Volume 5, Issue 4, pp.-439-444.

Copyright: Copyright@2013 Kina J.R., et al. This is an open-access article distributed under the terms of the Creative Commons Attribution License, which permits unrestricted use, distribution and reproduction in any medium, provided the original author and source are credited.

\section{Introduction}

The etiology of periodontal disease is multifactorial, in which bacterial plaque plays an essential role in the development of the disease [1-12]. Nevertheless, it has become clear that not all patients with periodontitis harbor the same subgingival microflora, and patients that harbor bacterial species recognized as indicators of periodontal disease may not develop periodontal breakdown all the time $[3,6,7,10,12-15]$. Periodontal disease onset and progression results from a multifactorial etiology and the etiologic agents must act in association to induce periodic and destructive acute inflammatory responses intercalated by a stable chronic inflammatory process $[5,10,11,14,15]$. Since bacteria are the essential etiologic agents that cause periodontitis, the principal goal of periodontal treatment is based on control or elimination of bacterial pathogens and theirs derived products $[1,3,4]$. The treatment procedures involve nonsurgical therapy that controls the growth of harmful bacteria and in advanced cases, surgical therapy $[1,16]$. However the exact moment to perform the procedure to treat periodontal disease is not well defined. Once periodontal disease is diagnosed, the actual stage of disease activity may not be precisely established [15]. The active acute phase of periodontal disease, which causes periodontal tissue destruction, is not persistent and does not produce the same pattern of destruction in the periodontal tissues around the teeth $[10,13-15,17,18]$. Instead, the active acute phase is brief, periodic, and may occur in various locations in different teeth or only in a single tooth, always intercalated by a stable chronic phase $[13,15]$. Due the absence of a predictive trustful test to identify earlier the destructive acute phase of periodontal disease, the majority of periodontal treatments are performed when the disease presents a stable and reparative chronic phase. To treat any disease the main goal should be to diagnose and to control precociously all etiologic factors. Specifically for periodontal disease, the procedures must be equal and subsequently closely monitor for signs of disease activity on a regular basis, to achieve the finest prognostic. In this investigation, the reparative aspects of diseased periodontal tissue were analyzed such as: (1) bone wall conditions inside untreated periodontal bone defects by using direct observation during periodontal surgery; (2) radiographic images obtained when a periodontal probe was inserted inside of the periodontal pocket; (3) reparative conditions in histologic images of untreated periodontal 
pockets identified in relevant publications.

Those aspects seem to be important to discuss the periodontal disease progression that until now present obscure feature, inducing toward an inaccurate moment to treat the disease.

\section{Materials and Methods \\ Periodontitis Subjects}

The experimental group consisted of 81 subjects ( 40 females and 41 males) 25-45 years old with untreated moderate-to-severe periodontitis who were randomly recruited from a private dental office, located in Araçatuba - São Paulo - Brazil. It was made clear to all potential subjects that participation was voluntary. All subjects were fully informed of the purpose of our research and the methodology, and signed an informed consent term for participation in the study.

Inclusion Criteria: subjects with periodontal disease were required to be $\geq 25$ years of age, have $\geq 25$ natural teeth. Criteria for periodontitis were a clinical attachment level measurement $\geq 5 \mathrm{~mm}$, bleeding upon probing (within 30s after probing) and radiographic evidence of periodontal bone loss, as a minimum in a side of the one tooth. Clinical attachment level was recorded as the distance from the cemento-enamel junction (CEJ) to the base of the clinical pocket. Clinical attachment level measurements were recorded only by an experienced periodontist (E.F.U.K.) using Michigan 0 periodontal probes with Willians markings.

Exclusion Criteria: subjects who had periodontal treatment in the previous 12 months or any medical condition that would require antibiotic prophylaxis for routine dental procedures. Individuals who had taken antibiotics in the previous 3 months or who were pregnant were also excluded from this study.

\section{Radiographic Images}

When clinical attachment level measurement was made in mesial or distal side of the each selected tooth, a radiographic image was obtained with the probe inserted inside of the periodontal pocket as contrast. The criteria to select mesial or distal surface, was the most significant clinical attachment loss. When the measurements were equal, the mesial side was selected. Only an experienced periodontist (E.F.U.K.) performed the evaluation, by applying in the probe a pressure-controlled vertical force until a resistance was felt, providing an objective and reproducible method to monitor the probe measurement [19].

The 81 subjects selected provided a sample of 133 diseased areas, which presented preestablished parameters. Periodontal bone defects were categorized by clinical status and not by types of architecture. Sixty-six of the periodontal defects were located in the maxilla and sixty-seven periodontal defects were in the mandible. Initial therapy was not applied before surgical procedure, to avoid interferences in the results.

\section{Surgical Procedure}

The surgical therapy applied was based on modified Widman flap surgery, an access flap operation indicated for the treatment of all types of periodontitis, especially to provide improved visual access to the periodontally involved tissues. The 81 subjects were scheduled for periodontal surgical treatment between March 2006 and April 2011. An experienced periodontist (J.R.K.) treated the 81 randomly selected subjects affected by periodontal disease. In each diseased area, the full mucoperiosteal buccal and lingual access flaps were raised. Granulation tissue and pocket epithelium were removed and the surgical area was rinsed with saline. Each perio- dontal bone defect was documented by digital photography. Manual instrumentation was carried out until no plaque or calculus was visible and all pathologically exposed root surfaces felt hard and smooth by using a fine explorer. The amount of cortical bone reparation was not relevant for this research due to the goal being verification of whether a reparative phase occurred after the destructive phase of periodontal disease. The mucoperiosteal flap was replaced and interrupted suture was made with silk suturing material (4/0 Ethicon, Johnson \& Johnson, Brazil). All periodontal treatments applied to the 81 subjects were satisfactorily completed and a specific maintenance program was established for each subject.

\section{Histologic Assessment by Literature Review}

Relevant publications in the English language, which presented histologic images of untreated periodontal pockets, were identified after Medline and PubMed (J.K., M.K. and T.Y.U.S.). Include criteria was histologic images of untreated periodontal pockets and exclude criteria was histologic images showing results of treatment applied in periodontal disease or histologic images of health periodontium. Length of the review period of the dental literature was not relevant, since all include criteria was observed. The search strategy was based in the following key words and MeSH terms: periodontal pocket/pathology; histologic periodontal pocket; periodontal disease; disease models; animal; periodontology books. The histologic images of untreated periodontal pockets were scanned, and then the presences of the gingival-attached connective tissue zone above the underlying destroyed alveolar bone were evaluated [2029].

\section{Results}

\section{Surgical Assessment}

According to the methodology applied in this study, the surgical assessment of all 133 selected sites which had a clinical attachment level measurement $\geq 5 \mathrm{~mm}$ showed periodontal bone defects presenting various architectures and cortical bone reparations at different levels inside periodontal bone defects [Fig-1].

\section{Radiographic Images}

All radiographic images showed a space between the tip of the periodontal probe and the underlying destroyed alveolar bone [Fig2].

\section{Histologic Assessment by Literature Review}

All relevant publications searched in this study have illustrated histologic images of untreated periodontal pockets, showing repaired gingival-attached connective zone presenting various degree of inflammatory infiltrates, which separate the underlying destroyed alveolar bone from the apical end of the pocket epithelium [20-29].

\section{Discussion}

The essential requirement for the treatment of any disease is knowledge of its etiology. Although many questions remain to be answered about the pathogenesis of periodontal disease, the main cause has been defined. The bacterial plaque in proximity to the gingival margin seems to be the essential agent, which plays a role in inducing gingival inflammation $[1-4,6-10,12]$. The body of evidence is consistent and stems from epidemiologic data, microbiologic research, immunologic studies, and clinical observations [1$4,6-10,12,30,31]$. The study of experimental gingivitis by the withdrawal of all forms of oral hygiene performed by Löe, et al [1] was a landmark in the research of the bacterial etiology of gingival margin 
inflammation. These workers showed the pathogenic potential of normal oral commensal bacteria to induce gingivitis. Nevertheless, epidemiologic studies have clearly demonstrated that not all forms of gingivitis progress to periodontitis. Consequently, plaque-induced gingivitis is considered necessary, but insufficient cause of periodontitis. In the experimental gingivitis study, virtually all participants developed some degree of gingival inflammation in response to plaque accumulation. However, gingivitis susceptibility varied considerably among the various individuals who harbored the similar bacterial complex. These data demonstrates that susceptibility to periodontal disease differs among diverse individuals and differs in various sites in the same individual, and sometimes differs among surfaces of a tooth $[1,5,10,13,15,17,32]$. This fact may be due the multifactorial etiology of periodontal disease that requires an association among bacteria and diverse risk factors to induce the development of periodontal disease destructive phase $[5,11,14,32]$.

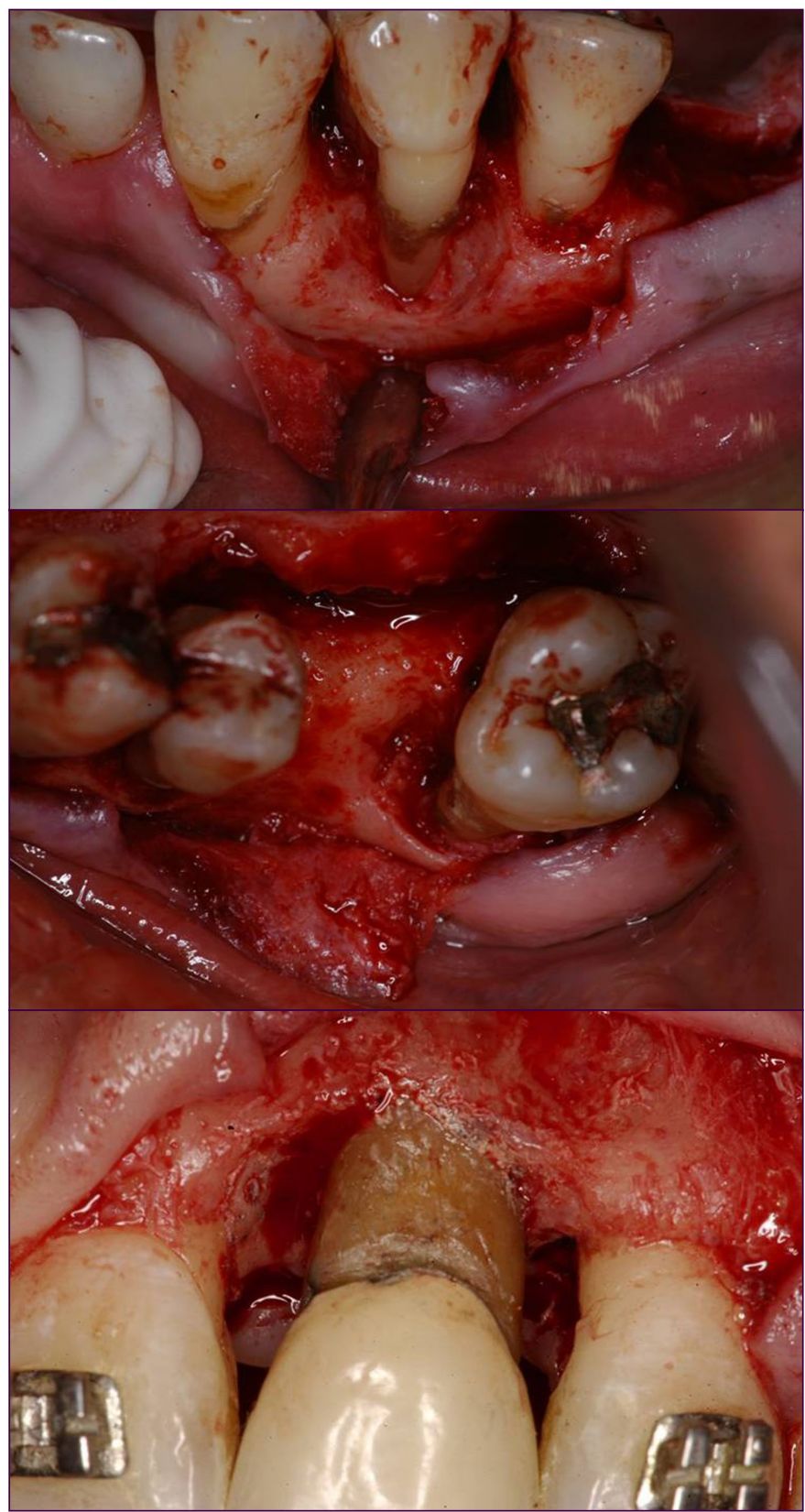

Fig. 1- Untreated bone defects showing cortical bone reparations at different levels

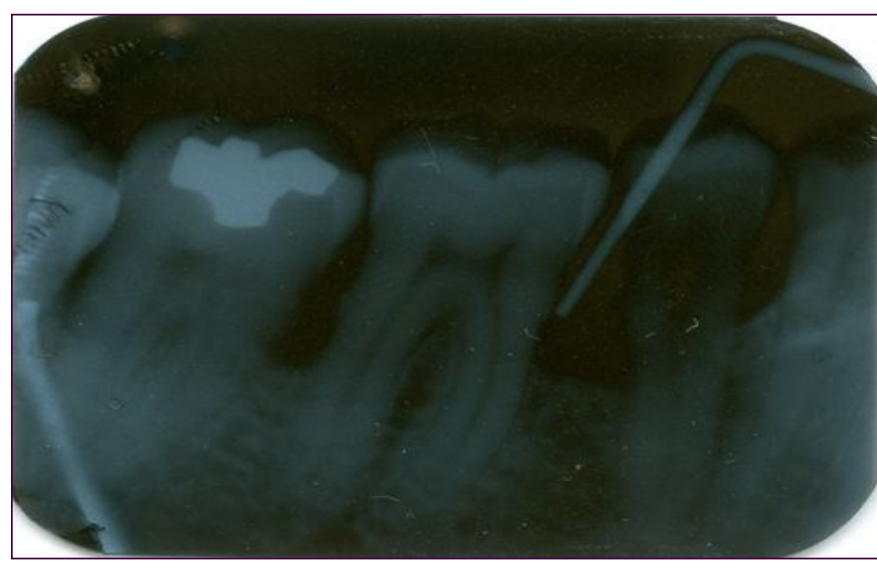

Fig. 2- Image showing a space between the tip of the periodontal probe and the underlying destroyed alveolar bone

The risk factor may be an inherent characteristic associated with an increased rate of a subsequently occurring disease, but does not necessarily cause the disease. In periodontal disease, the risk factors may be defined as local environmental factors, behavioral factors in nature and systemic factors, which may be responsible in providing an ideal environment for bacterial colonization and/or fragility in a determinate tooth or teeth and adjacent periodontal tissues and/or interference in the inflammatory process $[5,11,32$, 33]. Due to the association with risk factors, the opportunist endogenous bacteria, which are predominant in establishing gingival margin inflammation, also may induce destruction in the initial phase of periodontitis $[1,2,8,10,17]$. Periodontal disease could be considered as sequel of the inflammatory reaction, which must be always active, protecting individual against infection and possible septicemia, by bacteria present in the gingival sulcus, a critical area where junctional epithelium is an exclusive and fragile structure, separating connective tissue from an infected humid and warm oral environment. When risk factors interfere in the fragile equilibrium of the gingival sulcus defense, the opportunist bacteria may initialize periodontal tissue destruction by intense or deficient interaction with cells of the inflammatory process [34]. Periodontitis begins with microbial challenge, which induce a host-mediate response and destruction of periodontal tissue, caused by bursts of clastic cell activity, triggered by hyperactivated or primed polymorphonuclear leukocytes and factors generated during the inflammatory acute phase, such as eicosanoides and various proteins as enzymes that cause damage and rupture of the periodontium, promoting periodontal pocket establishment [17,34-37]. Periodontal pocket development is the most important clinical and pathologic alteration associated with inflammatory periodontal disease and may be considered as a risk factor for periodontal disease progression, by generating an anaerobic environment to be contaminated as a result of repeated infection by the various species or combination of the species as exogenous anaerobic and facultative bacteria $[3,6,7,9,10,12]$. These putative periodontal pathogens and their products may induce substantial pathological alterations, essentially in root surface exposed to the contaminated periodontal pocket. On the other side, due bacterial approximation to the ulcerated pocket epithelium, infected periodontal pocket also could be an infectious focus linked to the various systemic disorders, probably led by anachoresis, a process associated with dissemination of the microorganisms or/and toxics products into blood stream, assisting or causing infection in the various vital organs $[11,38-40]$. For this 
reason, it is extremely necessary to treat the contaminated periodontal pocket, by physical and chemical therapeutic periodontal methods, to prevent bacterial dissemination but not fundamentally to evict periodontal disease progression, since the profile of the periodontal disease activity is to maintain stability $[15,18]$. Thus, bacteria colonization is essential etiologic agent of periodontal disease and should be always under control but, to develop periodontitis seems to be necessary the association among opportunist commensal bacteria and other diverse risk factors. This fact may be corroborated for the evidence that the research which studied aggressive pathogenic bacteria establishing correlation with periodontitis, have proposed a methodology to harvest the aggressive pathogenic bacteria from recognized periodontally diseased sites. It is improbable that these exogenous anaerobic and facultative aggressive bacteria act intensively as the unique etiological factor, mainly for the onset of periodontitis [3-7,9-12,14,15,17]. In addition when researchers induce experimental periodontitis, the workers always needs to apply risk factors, such as a ligature around the teeth, to assist bacteria to provoke periodontal disease [4,19,27-29]. Furthermore, is impossible to identify in relevant publications, any consistent evidence confirming that pathogens considered as periodontopathogenic bacteria, could induce experimental periodontitis without association with an additional risk factor. Bacteria may not establish preference to colonize only one tooth or group of teeth $[1,5,10,13-15,32]$. However the vast majority of periodontal disease induce localized destruction in a dental region, around a single tooth, in an interproximal space, or on the side of a single tooth, fundamentally due association among opportunist bacteria and the diverse local risk factors, which may establish a favorable environment for bacteria colonization and development or/and may provoke fragility in periodontal tissues around a tooth facilitating bacterial action $[5,13,32,33,41]$. When periodontal destruction is generalized, probably opportunist bacteria are associated with systemic risk factors, which affect inflammatory process inducting altered defense response against opportunist bacteria and consequently, generalized periodontal destruction $[5,11,32,43]$. To prevent or to treat any disease always will be necessary to eliminate or to establish control in all etiologic factors or/and improve host local defense or/and general resistance against the entire etiologic factors, to promote homeostasis in diseased areas through a long stated period. It is almost impossible to establish for each precise tooth, an intrinsic optimum plaque control level, which even in association with the various possible risk factors, who may appear or disappear at any instant, could not induce destructive periodontal disease in a specific moment. The essence of periodontal therapy involves mainly the control of bacteria, but also should involve the control of risk factors to induce supplementary, predictable and constant periodontal stability. Bacterial plaque forms part of the natural microbial flora related to internal and external human body surfaces, and host factors operate in all cases. However, it is difficult to find generalized destruction caused by periodontal disease in all the teeth of the same individual $[10,13-15]$. When periodontal disease determines the localized supporting bone loss in one tooth, the relative composition of the bacterial flora in that established deep pocket are very different from those of the gingival sulcus in adjacent teeth $[3,6,12]$. Colonization with a specific periodontal pathogen seems necessary, but not sufficient for periodontal disease onset and progression since most colonized and untreated sites remain quiescent for long periods, regardless of various Gram-negative anaerobic or facultative bacteria species may themselves disrupt cells from host de- fense mechanism $[15,18,41]$. Additionally, when a periodontal pocket is produced, the physiologic occlusal force may act on dentition that is seriously weakened by the loss of supporting alveolar bone, resulting in another risk factor, which also may be important in the progression of periodontal disease [33,41]. Although the periodontal pocket development amplifies the quality and quantity of the bacteria and risk factors, which could maintain periodontal disease active and destructive, in the sequence of the destructive inflammatory acute phase a reparative inflammatory chronic process is expected to be established $[5,11,15,17,32]$. In fact, it is always possible to find in any untreated periodontal pocket, repair of the cementum and the gingival-attached connective tissue zone, which separates the underlying destructed alveolar bone from the apical end of the ulcerated periodontal pocket epithelium, despite evidence that inflammatory cells may infiltrate to various degrees into the repaired gingival-attached connective tissues [18,20-29]. In addition, during periodontal surgical treatment, it is very easy to find reparations of cortical bone at various levels inside the periodontal bone defects. Because of this reparative process, in order to augment healing of periodontal defects, several bone graft surgical procedures require repaired cortical bone and gingival-attached connective tissue mechanical debridement, to induce an intra-marrow and periodontal ligament mesenchymal stem cells penetration into periodontal bone defects. The repaired structures separate infected periodontal pockets from the intra-marrow alveolar bone/periodontal ligament and seem to be the main barrier against progression of the destructive periodontal disease. In this study, the surgical images showing a dental clear space between calculus and the underlying destroyed alveolar bone and the radiographic findings showing a space between the tip of the periodontal probe and the underlying destroyed alveolar bone, may corroborate the presence of repaired gingival ligament above destroyed alveolar bone, analyzed in histological images. Furthermore, several radiographic images obtained with periodontal probe inserted in mesial or distal side of the diseased teeth, as contrast, also showed radiopaque images corresponding to the thin layer of dense cortical bone inside periodontal bone defects, suggesting bone reparation in untreated periodontally diseased sites. All the evidences analyzed in this study suggested that periodontal disease is predominantly chronic, quiescent, showing reparative phases in different levels. Periodontitis is a complex multifactorial disease that progress by successive rapid and short active phases, always intercalated by slow and long quiescent phases, and for each successive active phase, arise a variation in the quantity and quality of the interrelationship among all probable etiologic agents. In addition, every individual and each distinct area of the same individual may develop in a determinate moment, specific temporary fragility and reaction for the same involved etiologic agents, which in association could be able to initiate or to cease or to restart periodontal disease progression. This transitory risk factor may be defined as coping behaviors, principally the stress, a physical, chemical and emotional risk factor inherent for each individual, who can come from any situation or thought, attacking in various aggressive levels, that can appear or disappear at any moment, which interfere in host defense mechanism and drastically in stomatognathic system, probably, may be one of the principal additional dispositional etiologic risk factor to trigger or to cease periodontal destruction [43]. Due periodontal disease characteristic, is very difficult to establish precise diagnosis for all etiologic agents and the momentary specific individual fragility, involved during periodontal disease progression $[5,11,15,18,32,33,41,42,43]$. This feature also 
could explain the absence of a predictive trustful biomarker test to identify earlier, the destructive acute phase of periodontal disease [37]. The main diagnostic methods applied to identify periodontal disease are periodontal attachment loss measurements, probing depth verification, and radiographic evaluation. By using these methods, the clinician may determine that at some time in the past, periodontal disease was active and destructive. In the majority of periodontal surgical procedures, the treatment is applied when the reparative phase is ongoing, promoting formation of cortical bone inside the periodontal defect and immediately above, the repair of the cementum and gingival-attached connective tissue zone [15]. Then, the most favorable moment to treat periodontal disease is still unclear, although periodontal surgery procedures may be useful to treat periodontal pockets a potential focus of infection, by controlling bacteria and their products, but are not able to control the multiplicity of the interaction among all etiologic agents and specific individual fragility, involved in the development of periodontal disease $[5,11,16,18,32,33]$. Researching to improve the diagnostic procedures may enhance knowledge about the complex interrelationships among the diverse etiologic factors involved along progression of periodontal disease, permitting treatment when or before the disease become active and destructive. The rate of bone loss in the inflammatory acute destructive phase is extremely rapid, but the host defense response and healing is quite impressive [17]. It is consistent with acute infections in other clinical situations, in which the more acute the exacerbation of the lesion, the more dramatic the host defensive response. The repair of injury begins as soon as tissue damage occurs. It seems to hold for the diagnostic and treatment in the destructive acute phase of the periodontal disease for the finest reconstitution of lost periodontal tissue, or immediately before periodontal destruction [44]. Regardless of the fact that usually periodontal disease is treated when it is stable, by controlling all possible etiologies as bacteria and predisposing risk factors, the sequel of its activity remain as an anatomic defect in the periodontium. The treatment of the periodontal disease sequel include surgical exposure of the area, debridement of the diseased site and the adjacent involved root surface, complementary root physical and chemical treatment, and subsequently the application of resective or regenerative procedures, via altering the periodontal bony defect morphology and then surgical closure of the area [16]. These procedures generally are effective in controlling the periodontal contamination by bacteria and theirs products but are unpredictable to recuperate periodontal destructed tissue at pre disease stage [45]. In the early stages of periodontal wound healing, the wound stabilization and nutrition may be a critical variable to achieve regeneration. All healing wounds proceed through into three phases during the process of healing, following injury: inflammation, fibroblasticgranulation, and matrix formation and remodeling [46-48]. In the sequencing of these events, during the early phase of healing, a fibrin clot is formed. In any wounds, this fibrin clot bridges the space between two vascular wound margins and serves as a base that epithelial cells migrate across to cover the wound, inducing protection to the underlying connective tissue as healing progresses. Periodontal surgical wounds follow a similar healing pattern, but there are some significant differences in this specific wound healing environment that may affect the outcome of the procedures. When periodontal wounds are closed and sutured, one of the wound margins is an avascular and rigid periodontitis-affected and altered root surface $[18,38]$. This detail induces a fibrin clot formation with a fragile initial attachment to the altered root surface, to prevent epithelial down growth and to form a scaffold for development of a cell and collagen fiber attachment mechanism [46-48]. Then a fibrin clot adherent to the altered root surface is a fragile but vital part of early periodontal wound healing. The fibrin clot must form and adhere to the altered root surface for adequate time to allow for proper wound maturation, including connective tissue formation and development, before a new connective tissue attachment can occur. If this first series of events is disrupted, or if the initial attachment of fibrin or/ and immature connective tissue is ruptured, then a pattern of healing including a long junctional epithelium to the base of the original periodontal pocket is expected to occur $[47,48]$. In periodontal surgery procedure the early wound healing stability is easily disturbed inducing a disruption in the fibrin clot, which is frail attached to the altered root surface. This occurrence allows in this unique healing site a communication between the underlying connective tissue and the contaminated, humid and warm oral environment as healing progresses. To prevent infection, epithelial proliferations extend apically on the tooth aspect, establishing a long junctional epithelium attached to the root surface by hemidesmosomes $[47,48,49]$. The long junctional epithelium is a fragile structural and functional adaptation enabled to produce a defensive biological mechanism, which is responsible to control the constant microbial challenge by isolating the exposed connective tissue in the inner surface of the wound from contaminated oral environment [49]. However, this fragile protective structure also effectively prevents connective tissue from gaining access to the root surface and precludes periodontal regeneration $[47,48]$. As well as in periodontal disease, dentoalveolar abscess occur when polymicrobial opportunistic pathogens resembling periodontopalhogenic bacterial flora, infect the dental pulp assisted by a predisposing factor as dental caries $[12,50]$. The infection induces pulpar necrosis, which extends through the root canal system of the tooth, progressing rapidly to involve the periapical structures. The development of infection in the periapical region, defines a localized area of inflammation arising in the periodontal ligament space, implicating in periodontal tissue contamination and destruction, similar as induced by periodontal disease [45]. However, when an endodontic therapy is done properly, the pathway that leads the periapical area to the contaminated oral cavity is obstructed. Without junctional epithelium interference, the periodontal periapical tissues as cementum, bone and periodontal ligament may regenerate, acquiring pre disease condition, even without any specific physical and chemical treatment on the periodontal periapical contaminated root surface.

\section{Conclusion}

Then the major factors inhibiting periodontal disease prevention, treatment and predictable regeneration appears to be: (1) The multifactorial etiology of periodontal disease and the distinct and variable host reaction for the same etiologic agents; (2) the nature and complexity of marginal periodontal tissue around the teeth, the unique tissue in the body, structured into the basic components of cementum, alveolar bone and periodontal and gingival ligament, which are separated from an infected, humid and warm oral environment by a fragile junctional epithelium.

\section{References}

[1] Löe H., Theilade E. and Jensen S.B. (1965) J. Periodontol., 36, 177-187.

[2] Theilade E., Wright W.H., Jensen S.B. and Löe H. (1966) J. Periodontal. Res., 1, 1-13. 
[3] Socransky S.S. (1970) J. Dent. Res., 49, 203-222.

[4] Lindhe J., Hamp S. and Löe H. (1973) J. Periodontal. Res., 8, 1 $-10$.

[5] Pennel B.M. and Keagle J.G. (1977) J. Periodontol., 48, 517532.

[6] Socransky S.S. (1977) J. Periodontol., 48, 497-504.

[7] Slots J. (1979) J. Clin. Periodontol., 6, 351-382.

[8] Moore W.E., Holdeman L.V., Smibert R.M., Good I.J., Burmeister J.A., Palcanis K.G. and Ranney R.R. (1982) Infect. Immun., 38, 651-667.

[9] Moore W.E. (1987) J. Periodontal. Res., 22, 335-341.

[10]Dzink J.L., Socransky S.S. and Haffajee A.D. (1988) J. Clin. Periodontol., 15, 316-323.

[11]Heitz-Mayfield L.J.A. (2005) J. Clin. Periodontol., 32, 196-209.

[12]Socransky S.S. and Haffajee A.D. (2005) Periodontol 2000, 38, 135-187.

[13]Ormes W.M.Jr. and Sheridan R.C.Jr. (1965) J. Periodontol., 36, 112-114.

[14]Goodson J.M., Tanner A.C., Haffajje A.D., Sornberger G.C. and Socransky S.S. (1982) J. Clin. Periodontol., 9, 472-481.

[15]Socransky S.S., Haffajee A.D., Goodson J.M. and Lindhe J. (1984) J. Clin. Periodontol., 11, 21-32.

[16]Ramfjord S.P. and Nissle R.R. (1974) J. Periodontol., 45, 601607.

[17]Craig R.G., Yip J.K., So M.K., Boylan R.J., Socransky S.S. and Haffajee A.D. (2003) J. Periodontol., 74, 1007-1017.

[18]Kina J.R., Kina J., Kina E.F., Kina M. and Soubhia A.M. (2008) J. Appl. Oral. Sci., 16, 205-208.

[19]Van der Velden V. and Jansen J. (1981) J. Clin. Periodontol., 8 (2), 107-116.

[20]Glickman I. and Smulow J.B. (1974) Periodontal Disease: Clinical, Radiographic and Histopathologic Features, W.B. Saunders, Philadelphia.

[21]Glickman I. and Carranza F.A. (1979) Glickman's Clinical Periodontology: Prevention, Diagnosis and Treatment of Periodontal Disease in the Practice of General Dentistry, W.B. Saunders, Philadelphia.

[22]Grant D.A., Stern I.B. and Listgarten M.A. (1988) Periodontics in the Tradition of Gottlieb and Orban, Mosby, St. Louis.

[23]Lindhe J. (1989) Textbook of Clinical Periodontology, 2nd ed., Munksgaard, Copenhagen.

[24]Carranza Jr.F.A. (1990) Glickman's Clinical Periodontology, W.B. Saunders, Philadelphia.

[25]Genco R.J., Goldman H.M. and Cohen D.W. (1990) Contemporary Periodontics, C.V. Mosby, St. Louis.

[26]Bartold P.M. and Narayanan A.S. (1998) Biology of the Periodontal Connective Tissues, Quintessence, Chicago.

[27]Niikura K. (2006) J. Periodontol., 77, 1211-1216.

[28]Seto H., Toba Y., Takada Y., Kawakami H., Ohba H., Hama H., Horibe M. and Nagata T. (2007) J. Periodontal. Res., 42, 85-89.

[29]Shoji K., Ohtsuka-Isoya M., Shimauchi H. and Shinoda H. (2007) J. Periodontol., 78, 152-156.

[30]Nisengard R.J. (1977) J. Periodontol., 48, 505-516.
[31]Borrell L.N. and Papapanou P.N. (2005) J. Clin. Periodontol., $32,132-158$.

[32]Genco R.J. (1996) J. Periodontol., 67(10), 1041-1049.

[33]Nyman S., Lindhe J. and Ericsson I. (1978) J. Clin. Periodontol., 5, 213-225.

[34]Dennison D.K. and Van Dyke T.E. (1997) Periodontol. 2000, 14, 54-78.

[35]Takata T. and Donath K. (1988) J. Periodontol., 59, 215-221.

[36]Smalley J.W. (1994) Adv. Dent. Res., 8, 320-328.

[37]Kina J.R., Yoshida N., Goseki M., Sasaki S. and Ishikawa I. (1995) Bull. Tokyo Med. Dent. Univ., 42, 57-65.

[38]Karring T., Nyman S. and Lindhe J. (1980) J. Clin. Periodontol., 7, 96-105.

[39]Friedewald V.E., Kornman K.S., Beck J.D., Genco R., Goldfine A., Libby P., Offenbacher S., Ridker P.M., Van Dyke T.E. and Roberts W.C. (2009) J. Periodontol., 80, 1021-1032.

[40]Scannapieco F.A. (1998) J. Periodontol., 69, 841-850.

[41]Polson A.M., Meitner S.W. and Zander H.A. (1976) J. Periodontal. Res., 11, 290-298.

[42]Zambon J.J. (1985) J. Clin. Periodontol., 12(1), 1-20.

[43]Rosania A.E., Low K.G., Mc Cornick C.M. and Rosania D.A. (2009) J. Periodontol., 80, 260-266.

[44]Prichard J.F. (1953) Oral Surg. Oral Med. Oral Pathol., 6, 474482.

[45]Polson A.M. (1994) Periodontal Regeneration: Current Status and Directions, Quintessence, Chicago.

[46]Clark R.A.F. (1993) Invest. Dermatol., 11, 647-661.

[47]Polimeni G., Xiropaidis A.V. and Wikesjö U.M. (2006) Periodontol. 2000, 41, 30-47.

[48]Wikesjö U.M.E., Nilvéus R.E. and Selvig K.A. (1992) J. Periodontol., 63, 158-165.

[49]Bosshardt D.D. and Lang N.P. (2005) J. Dent. Res., 84, 9-20.

[50]Broak I., Franzier E. and Gher M. (1991) Oral Microbial. Immunol., 6, 123-125. 